\title{
Correlation between DNA ploidy by flow cytometry and chromosome 3 aberration in oral squamous cell carcinoma
}

\author{
JOERG HEMMER $^{1,2}$, KLAUS KRAFT $^{3}$ and WILLIE F.P. VAN HEERDEN ${ }^{1}$ \\ ${ }^{1}$ Department of Oral Pathology and Oral Biology, University of Pretoria, Pretoria 0001, \\ South Africa; ${ }^{2}$ Department of Tumor Biology, University of Ulm, D-89081 Ulm; \\ ${ }^{3}$ Department of Pathology, Military Hospital Ulm, D-89070 Ulm, Germany
}

Received July 6, 2005; Accepted August 22, 2005

\begin{abstract}
Although flow cytometric DNA ploidy has turned out as a significant predictor of survival in oral squamous cell carcinoma, little is known about the underlying karyotypic structure of gross aneuploidy. We therefore analysed one diploid and 9 aneuploid carcinomas with relative DNA contents between 1.1 and 2.8 by fluorescence in situ hybridization with topologic markers for the centromere (3cen) and the terminal regions $(3 p, 3 q)$ of chromosome 3 . Progressing deviation of aberrant DNA contents from the normal diploid value correlated with increasing 3 cen copy numbers per cell. A pronounced marker heterogeneity suggested that DNA-aneuploid cell populations consisted of karyotypically different clones. Monosomy of 3p was the only chromosomal alteration in the DNA diploid tumour. A significant 3p underrepresentation was a recurrent finding also in 7 of 9 aneuploid carcinomas while a subset of cells in each of 2 other cases showed a complete loss of one sister chromosome 3 . In contrast, 7 of 9 aneuploid tumours exposed corresponding $3 q$ and 3 cen copy numbers, 2 showed a substantial $3 q$ overrepresentation. It appears that amplification of chromosome 3 plays a role in the development of aneuploidy and the concurrent overexpression of $3 \mathrm{q}$ target genes. Acquired loss of the short arm of chromosome 3 in DNA-diploid tumour cells may contribute to the manifestation of recurrent $3 q$ deletions in aneuploid cell populations.
\end{abstract}

\section{Introduction}

While a multistep carcinogenetic process, in which mutations of tumour suppressor genes and oncogenes may play a critical role, has been proposed for the development of oral squamous cell carcinoma (1), DNA flow cytometric studies provided evidence that gross karyotype rearrangements contribute to

Correspondence to: Professor Joerg Hemmer, Department of Tumour Biology, University of Ulm, Albert-Einstein-Allee 11, D-89081 Ulm, Germany

E-mail: joerg.hemmer@medizin.uni-ulm.de

Key words: oral cavity cancer, DNA flow cytometry, aneuploidy, chromosome 3 the acquisition of invasive and metastatic behaviour $(2,3)$. Aneuploidy has actually turned out as an independent predictor of survival in oral squamous cell carcinoma (3-6). The outcome of patients is essentially determined by a several-fold increase in risk of local and regional recurrence after the emergence of DNA-aneuploid cell populations (4,6-9).

DNA content aberrations signify a fundamental reorganisation of the tumour cell genome in which gains and losses of chromosomes substantially contribute to the expression of altered DNA contents (10). Remarkably, although DNA contents vary in wide range, thus implying corresponding karyotypic disparities, neither the metastatic behaviour of tumours nor the outcome of patients correlate with the degree of aneuploidy $(2,7)$. A conceivable explanation is that aneuploid clones, despite their overall differences in chromosomal composition, may share discrete karyotypic aberrations that are decisive for the expression of malignant behaviour. As multiple studies suggested that mutations involving the short arm of chromosome 3 may be critical for the progression of squamous cell carcinomas of the head and neck (11-19), we analysed the pattern and frequency of rearrangements of chromosome 3 by fluorescence in situ hybridization in oral squamous cell carcinomas with different degree of DNA ploidy.

\section{Patients and methods}

Ten patients with primary squamous cell carcinomas of the oral cavity were selected for this study. Fresh tissue samples were collected from resected tumours and were immediately cut in small pieces of approximately $1 \mathrm{~mm}$ in diameter. The samples were divided into two parts which either were processed for DNA flow cytometry or for fluorescence in situ hybridization (FISH).

For flow cytometry, the tissue pieces were thoroughly homogenized in $0.9 \% \mathrm{NaCl}$ by mincing with surgical scalpels. The nuclei were extracted by incubation in $0.5 \%(\mathrm{w} / \mathrm{v})$ acid pepsin solution ( $\mathrm{pH} 1.5$ ) with careful stirring for $5 \mathrm{~min}$, strained through a $50 \mu \mathrm{m}$ nylon mesh, and fixed with $70 \%$ ethanol. The nuclei were stained with $5 \mathrm{ml}$ of a DNA specific staining solution containing $0.2 \mathrm{mg}$ 4',6-diamidino-2-phenyl-indole (DAPI, Serva, Heidelberg, Germany) and $11.8 \mathrm{~g}$ citric acid trisolium salt dihydrate dissolved in $100 \mathrm{ml}$ distilled water ( $\mathrm{pH} 8.0$ ). The minimum incubation time was $30 \mathrm{~min}$ at room 

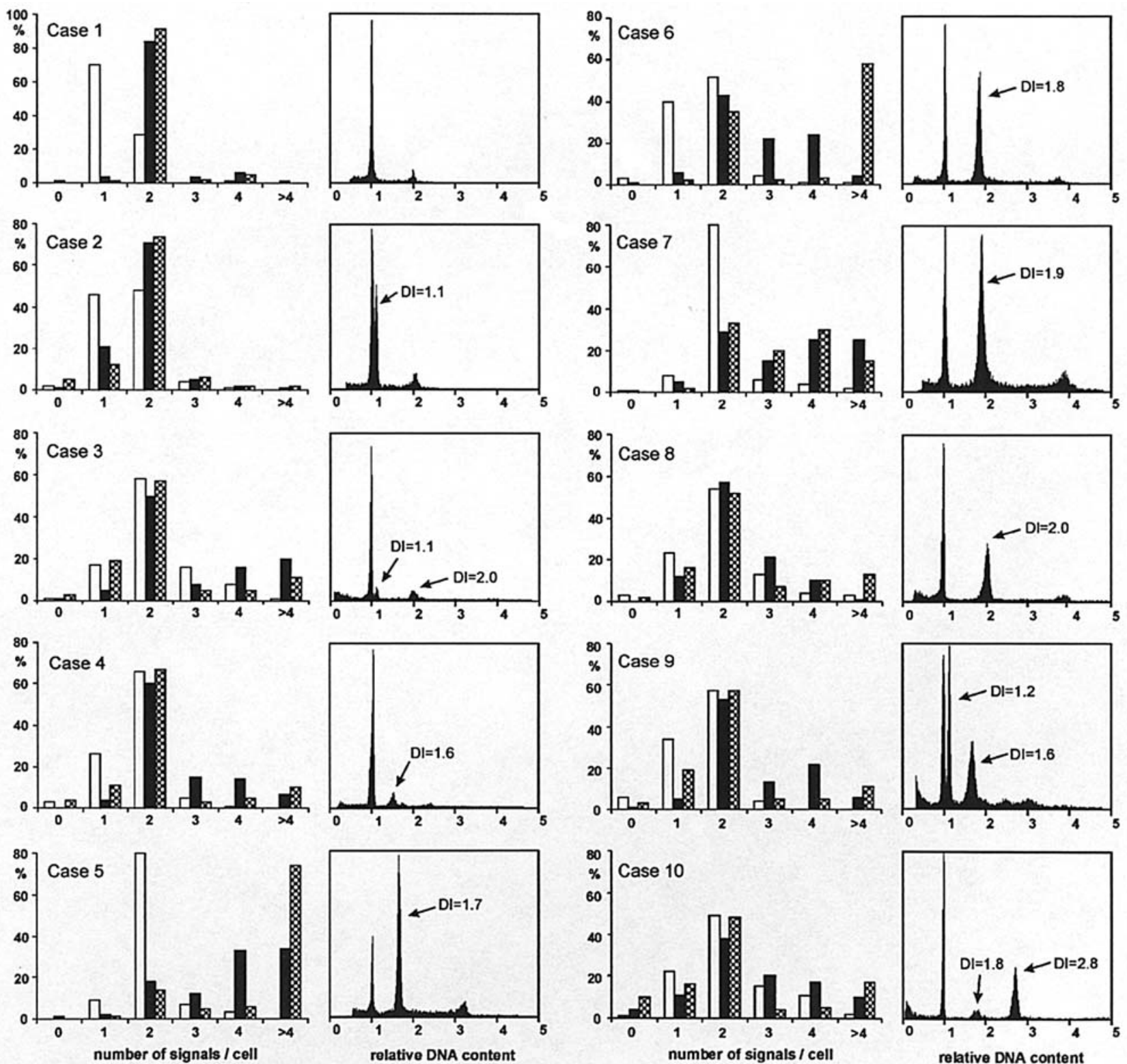

Figure 1. Frequency distribution of topologic DNA markers for chromosome 3 and corresponding DNA histograms. Left columns (white), $3 p$ terminal marker; central columns (black), $\alpha$ satellite marker; right columns (dotted), $3 q$ terminal marker; DI, DNA index.

temperature. Flow cytometry was carried out using a PAS II flow cytometer equipped with a high-pressure $100 \mathrm{~W}$ mercury lamp (Partec, Münster, Germany). The diploid cell population was used as a reference standard for the identification of aneuploid clones. The DNA index was expressed by the quotient of the respective modal peak values and reflected the discrepancy between the aneuploid DNA content and the normal diploid value (DNA index $=1.0$ ).

For FISH, nuclei were extracted by mincing the tissue samples with surgical scalpels in $2.5 \%(\mathrm{w} / \mathrm{v})$ citric acid. Tissue fragments were removed using a $50 \mu \mathrm{m}$ nylon mesh. The suspension was then passed through a Dounce homogenizer with a piston to vessel distance of $200 \mu \mathrm{m}$. Nuclei were sedimented by sucrose centrifugation, fixed in cold $3: 1$ methanol-acetic acid and stored at $-20^{\circ} \mathrm{C}$. Later, the fixed nuclei were spun onto silane-coated slides and air dried. After incubation in 2X SSC buffer (Sigma, Deisenhofen, Germany) for $30 \mathrm{~min}$ at $37^{\circ} \mathrm{C}$, the slides were dehydrated in ascending ethanol and air dried. The slides were incubated in $70 \%$ formamide $/ 2 \mathrm{X} \mathrm{SSC}$ for $2 \mathrm{~min}$ at $70^{\circ} \mathrm{C}$ and carried through an ascending $-20^{\circ} \mathrm{C}$ alcohol series. Digoxigeninlabeled probes specific for the tandem repeats of $\alpha$ satellite DNA located in the centromeric region of chromosome 3 (3cen) as well as for telomere specific repeat units of the 3 p26 and the 3q29 terminal ends of the chromosome (OncorAppligene, Heidelberg, Germany) were used for in situ hybridization. A solution was prepared containing $1.5 \mu \mathrm{l}$ of DNA probe and $30 \mu 1$ of $65 \%$ formamide/2X SSC/10\% $(\mathrm{w} / \mathrm{v})$ dextran sulphate. After $5 \mathrm{~min}$ incubation at $70^{\circ} \mathrm{C}$, the hybridization solution was placed onto the sample slides, coverslipped, and hybridized overnight at $37^{\circ} \mathrm{C}$. Hybridization was visualized using FITC-conjugated anti-digoxigenin Fab fragments (Roche Diagnostics, Mannheim, Germany). The slides were counterstained with DAPI. An average number of 201 nuclei (range: 110-537) was examined by fluorescence microscopy. 


\section{Results}

The 10 oral carcinomas had a wide range of DNA content aberrations (Fig. 1). One tumour consisted exclusively of flow cytometrically diploid cells, 9 carcinomas contained additional cell populations with atypical DNA contents. Six of aneuploid tumours expressed a single aneuploid cell line; 3 composed of 2 different aneuploid clones. The coefficients of variation, which reflects the sensitivity of flow cytometric measurements, ranged between $0.94 \%$ and $2.75 \%$ with a mean value of $1.81 \%(\mathrm{SD}=0.51)$.

For the DNA-diploid carcinoma (case 1), corresponding disomies were found for both the $\alpha$ satellite (3cen) and the $3 \mathrm{q}$ telomere specific DNA probes. While $30 \%$ of cells showed disomal findings for the $3 p$ telomere marker, an analogous proportion of $70 \%$ of cells was monosomal for that region. Non-disomal 3cen signal numbers were distinctive for DNA-aneuploid carcinomas. One tumour with near-diploid DNA content (case 2) contained cells with 3cen monosomy. Regardless of a 3cen signal number heterogeneity, there was an overall increase of 3cen copy numbers with progressing deviation of aberrant DNA contents from the normal diploid value. Except for cases 8 and 10, in which consistent monosomies of all topologic markers in a subset of cells suggested the complete loss of one sister chromosome 3, all other aneuploid tumours exposed a significant underrepresentation of $3 p$ copy numbers if compared to the frequency distribution of 3 cen signals (Kolmogorov-Smirnoff test, $\mathrm{P}<0.01$ ). While monosomies and disomies were prevailing findings for the $3 p$ region, the $3 q$ marker frequency did not differ from that of the centromere region, except cases 5 and 6 which showed a significant $3 q$ overrepresentation with up to 10 copy numbers per cell.

\section{Discussion}

The current study illustrated that chromosome 3 was involved in manifold structural and numerical rearrangements particularly in DNA-aneuploid oral squamous cell carcinomas. A distinct heterogeneity of topologic DNA markers suggested that aneuploid cell populations frequently composed of karyotypically different tumour cell populations. However, there was an overall association between 3cen marker polysomy and the degree of flow cytometric aneuploidy. Despite the gain in chromosome 3cen copy numbers during aneuploidy formation, there was a concurrent underrepresentation of $3 p$ signals per cell in 7 of 9 aneuploid tumours. Two other cases contained cells with a complete loss of one sister chromosome 3 . Monosomy of $3 \mathrm{p}$ was the only chromosome 3 aberration also in the DNA-diploid carcinoma.

These results are in agreement with reports showing that deletions involving the short arm of chromosome 3 , also observed as loss of heterozygosity in molecular genetic studies, are frequent cytogenetic changes in carcinomas of the head and neck $(11-16,18)$. Deletions of $3 p$ have been described for many kinds of solid tumours and gave reason to define a fragile site at $3 \mathrm{p} 14$, FRA3B, which is among the most common regions impacted by chromosomal strand breaks within the human genome (20). According to the concept that loss of tumour suppressor gene function is regarded as crucial for malignant transformation and tumour progression, the short arm of chromosome 3 is a prime candidate region to search for negative regulators of cell proliferation and differentiation. The number of tumour suppressor genes located on $3 p$ is hard to estimate, but the fragile histidine triad gene (FHIT) has turned out to play a significant role in oral carcinoma development (1).

Allelic loss of $3 p$ has frequently been detected in premalignant oral lesions and thus appears to be an early event in oral carcinogenesis $(16,21,22)$. Accordingly, $3 p$ marker monosomy in a flow cytometrically diploid tumour suggests a manifestation of $3 p$ deletions already at the DNAdiploid stage of oral squamous cell carcinoma progression. Amplification of chromosomes with acquired $3 p$ deletions during gross aneuploidy formation may actually contribute to the overall $3 p$ underrepresentation in aneuploid tumours.

In contrast, concordant $3 \mathrm{q}$ and 3 cen marker frequencies suggest a simultaneous amplification of the long arm of chromosome 3 with its centromere region in most of the aneuploid carcinomas. There were only two cases expressing a distinct $3 \mathrm{q}$ marker overrepresentation. These findings are consistent with analogous observations in head and neck carcinomas using comparative genomic hybridization (14). However, it is obvious that regulatory genes are subject to alterations of their dosage independent of whether the gain of genetic material is due to amplifications of discrete DNA sequences or to the increase in chromosome copy numbers. It has actually been evidenced that overexpression of p63, a p53 homologue located at 3q27-3q29, may play a critical role in squamous cell carcinoma development (23).

In conclusion, structural rearrangements involving the long arm of chromosome 3 are infrequent in oral squamous cell carcinomas, but the regular gain of $3 q$ copy numbers during gross aneuploidy formation may give rise to overexpression of target genes. Cell populations with $3 q$ deletions despite the overall gain in chromosome 3 copy numbers are regular characteristics of aneuploid tumours. Loss of $3 q$ in a DNA-diploid case may also suggest that deletions of the short arm of chromosome 3 precede the emergence of tumour cell populations with gross karyotype aberrations and may contribute to oral tumour progression.

\section{References}

1. Williams HK: Molecular pathogenesis of oral squamous carcinoma. Mol Pathol 53: 165-172, 2000

2. Hemmer J and Schön E: Cytogenetic progression in oral carcinoma: a DNA flow cytometric study on 317 cases. Int J Oncol 3: 635-640, 1993.

3. Hemmer J, Nagel E and Kraft K: DNA aneuploidy by flow cytometry is an independent prognostic factor in squamous cell carcinoma of the oral cavity. Anticancer Res 19: 1419-1422, 1999.

4. Chen RB, Suzuki K, Nomura T and Nakajima T: Flow cytometric analysis of squamous cell carcinomas of the oral cavity in relation to lymph node metastasis. J Oral Maxillofac Surg 51: 397-401, 1993.

5. Rubio Bueno P, Naval Gias L, Garcia Delgado R, Domingo Cebollada J and Diaz Gonzalez FJ: Tumor DNA content as a prognostic indicator in squamous cell carcinoma of the oral cavity and tongue base. Head Neck 20: 232-239, 1998.

6. Hemmer J, Kraft K and Kreidler J: The significance of DNA flow cytometry in predicting survival and delayed clinical manifestation of occult lymph node metastasis to the untreated neck in patients with oral squamous cell carcinoma. J Craniomaxillofac Surg 26: 405-410, 1998. 
7. Hemmer J, Kreidler J, van Heerden WFP, Raubenheimer EJ and Schön E: Flow cytometric cellular DNA content and lymph node metastasis in squamous cell carcinoma of the oral cavity. Int J Oncol 6: 1237-1242, 1995.

8. Hemmer J, van Heerden WFP, Raubenheimer EJ, Kreidler J and Schön E: Flow cytometric DNA ploidy and recurrence development in squamous cell carcinoma of the oral cavity. Int J Oncol 8: 113-116, 1996.

9. Hemmer J, Thein T and van Heerden WF: The value of DNA flow cytometry in predicting the development of lymph node metastasis and survival in patients with locally recurrent oral squamous cell carcinoma. Cancer 79: 2309-2313, 1997.

10. Hemmer J and Hauser C: Chromosomal composition of aneuploid clones with different DNA contents in head and neck squamous cell carcinomas as determined by combined flow cytometry and fluorescence in situ hybridization. Anal Cell Pathol 20: 197-203, 2000.

11. Kok K, Naylor SL and Buys CH: Deletions of the short arm of chromosome 3 in solid tumors and the search for suppressor genes. Adv Cancer Res 71: 27-92, 1997.

12. Latif F, Fivash M, Glenn G, et al: Chromosome 3p deletions in head and neck carcinomas: statistical ascertainment of allelic loss. Cancer Res 52: 1451-1456, 1992.

13. Maestro R, Gasparotto D, Vukosavljevic T, Barzan L, Sulfaro S and Boiocchi M: Three discrete regions of deletion at $3 p$ in head and neck cancers. Cancer Res 53: 5775-5779, 1993.

14. Bockmuhl U, Schwendel A, Dietel M and Petersen I: Distinct patterns of chromosomal alterations in high- and low-grade head and neck squamous cell carcinomas. Cancer Res 56: 5325-5329, 1996.

15. Uzawa N, Akanuma D, Negishi A, Iwaki H, Uzawa Y, Amagasa $T$ and Yoshida MA: Homozygous deletions on the short arm of chromosome 3 in human oral squamous cell carcinomas. Oral Oncol 37: 351-356, 2001.
16. Chakraborty SB, Dasgupta S, Roy A, Sengupta A, Ray B, Roychoudhury S and Panda CK: Differential deletions in $3 p$ are associated with the development of head and neck squamous cell carcinoma in Indian patients. Cancer Genet Cytogenet 146: 130-138, 2003.

17. Arai K, Shibahara T, Yamamoto $\mathrm{N}$ and Noma $\mathrm{H}$ : The presence of candidate tumor suppressor gene loci at chromosome $3 \mathrm{p}$ for oral squamous cell carcinomas. Oral Oncol 38: 763-771, 2002.

18. Jin Y, Mertens F, Mandahl N, et al: Chromosome abnormalities in eighty-three head and neck squamous cell carcinomas: influence of culture conditions on karyotypic pattern. Cancer Res 53: 2140-2146, 1993

19. Scully C, Field JK and Tanzawa H: Genetic aberrations in oral or head and neck squamous cell carcinoma 2: chromosomal aberrations. Oral Oncol 36: 311-327, 2000.

20. Matsuyama A, Croce CM and Huebner K: Common fragile genes. Eur J Histochem 48: 29-36, 2004.

21. Mao L, Lee JS, Fan YH, et al: Frequent microsatellite alterations at chromosomes 9p21 and 3p14 in oral premalignant lesions and their value in cancer risk assessment. Nat Med 2: 682-685, 1996.

22. Partridge M, Emilion G, Pateromichelakis S, A'Hern R, Phillips E and Langdon J: Allelic imbalance at chromosomal loci implicated in the pathogenesis of oral precancer, cumulative loss and its relationship with progression to cancer. Oral Oncol 34: 77-83, 1998.

23. Di Como CJ, Urist MJ, Babayan I, et al: p63 expression profiles in human normal and tumor tissues. Clin Cancer Res 8: 494-501, 2002. 